\title{
Discrete Time Point-to-Point Control of Flexible Structures
}

\author{
N. Al-Masoud ${ }^{1}$ Shih-Yu Chu ${ }^{2}$ Tarunraj Singh ${ }^{3}$ \\ School of Engineering \\ SUNY at Buffalo, Buffalo, New York, 14260
}

\begin{abstract}
The primary objective of this work is to investigate linear time invariant systems undergoing rest to rest maneuvers in a finite time using the discrete time domain approach. Using a given sampling period, the governing equations of linear systems are first discretized into the equivalent discrete time domain representation. To decouple the resulting difference equations, the system equations are converted into the Jordan Canonical Form by using a similarity transformation. The decoupled Jordan Canonical equations are converted to a set of algebraic input/output equations with embedded end-points conditions, by a recursive approach. The optimal or sub-optimal control profiles required to achieve the desired maneuver can be easily calculated through basic manipulation. The sensitivity of the design to the uncertainities in the system parameters is reduced by introducing sensitivity equations, and the design is found to be robust to these uncertainities.
\end{abstract}

\section{Introduction}

The control of physical systems with digital computers is becoming more popular in industry. Many new digital control applications are being stimulated by microprocessor technology with applications such as automobiles and household appliances. Among the advantages of the digital logic for control are the increased flexibility of the control programs and the decision-making or logic capability of digital systems. Systems requiring a change in state in a finite time are often referred to as point to point control problems. Practical examples of this class of problems range from control of large space apparatus (Juang [1]),(Singh [2]), robotic manipulators (Cannon [3]) and small scale apparatus like computer disk drives where precise point to point positioning is required (Bhat [4]). A linear time invariant system with one rigid mode and one flexible mode is considered in this work to illustrate the proposed technique. The governing equations of motion of

\footnotetext{
${ }^{1}$ Graduate Student, Mechanical \& Aerospace Engineering

${ }^{2}$ Ph.D. Candidate, Civil, Structural, \& Env. Engineering

${ }^{3}$ Associate Professor, Mechanical \& Aerospace Engineering
}

the system are transformed from continuous time domain to discrete time domain with different sampling periods. The system differential equations are decoupled using similarity transformation resulting in a set of algebraic equations. The resulting system is first solved assuming sub-optimal polynomial control profile at different sampling periods. To achieve an optimum control sequence $u_{i}^{*}, u_{i+1}^{*}, \ldots, u_{N-1}^{*}$, the Lagrange multipliers approach and the Hamiltonian at each interval of interest $[i, N]$ is used to minimize the performance index which is chosen to be the power consumed .

\section{Problem Formulation}

In point-to-point control problems, the vector $y(t)$ is completely specified at two different instants of time, $t_{1}$ and $t_{2}$, and the control input $\underline{u}(t)$ required to achieve these end conditions is to be determined. Let $N=\left(t_{2}-\right.$ $\left.t_{1}\right) / T$ be the total sampling interval, and the boundary conditions at the beginning and end of maneuver are specified as $y\left(t_{1}\right)=y(0)$ and $y\left(t_{2}\right)=y(N T)$. The behavior of this system can be characterized by a set of one or more differential equations. If the system is linear and time-invariant, the dynamic equations can be written in a state variable form as

$$
\underline{\dot{y}}(t)=A \underline{y}(t)+B \underline{u}(t)
$$

The solution of Eq. (1) is

$$
\underline{y}(t)=\Phi\left(t-t_{0}\right) \underline{y}\left(t_{0}\right)+\int_{t_{0}}^{t} \Phi(t-\tau) B \underline{u}(\tau) d \tau
$$

where, $\underline{y}\left(t_{0}\right)$ is the initial state. The input $\underline{u}(\tau)$ is constant between any two consecutive sampling instants based on the assumption of zero-order hold $(Z O H)$, that is $\underline{u}(\tau)=\underline{u}(k \Delta T)$, for $k \Delta T \leq \tau<(k+1) \Delta T$. By substituting $t_{0}=k \Delta T$, Eq. (2) becomes

$\underline{y}(t)=\Phi(t-k \Delta T) \underline{y}(k \Delta T)+\int_{k \Delta T}^{t} \Phi(t-\tau) B d \tau \underline{u}(k \Delta T)$

Eq. (3) describes the state vector $y(t)$ at all times between the sampling instants $k \Delta \bar{T}$ and $(k+1) \Delta T$, for $k=0,1,2, \ldots, \mathrm{N}-1$ (for simplicity, $T$ will replace 
$\Delta T)$. For numerical simulations, it is more convenient to describe $y(t)$ only at the sampling instants. Let $t=(k+1) T$, then Eq. (3) becomes the discrete state equations of the sampled-data system.

$$
\underline{y}[(k+1) T]=\mathbf{A}_{\mathrm{d}} \underline{y}(k T)+\mathbf{B}_{\mathrm{d}} \underline{u}(k T)
$$

where, $\mathbf{A}_{\mathbf{d}}=\Phi(T)=e^{A T}$ is the state transition matrix of $\mathbf{A}$, and $\mathbf{B}_{\mathbf{d}}=\Theta(T)=\int_{0}^{T} \Phi(T-\tau) B d \tau$ is the discrete control influence matrix. The most straightforward method of solving Eq. (4) under the boundary constraints is by recursion (Kuo [6]). Using the transition

matrix property $\Phi(N T)=\overbrace{\Phi(T) \Phi(T) \cdots \Phi(T)}^{N \text { terms }}=\mathbf{A}_{d}^{N}$, the solution of Eq. (4) is

$$
\underline{y}(N T)=\mathbf{A}_{\mathbf{d}}{ }^{N} \underline{\mathrm{y}}(0)+\sum_{k=0}^{N-1} \mathbf{A}_{\mathbf{d}}{ }^{N-k-1} \mathbf{B}_{\mathbf{d}} \underline{u}(k T)
$$

Assuming full controllability, a unique transformation can be found such that the discrete state equations Eq. (4), with some rearrangement, can be re-written in Jordan canonical form (Miu [5]).

$$
\underline{x}[(k+1) T]=\mathbf{J} \underline{x}(k T)+\Gamma \underline{u}(k T)
$$

where, $\mathbf{J}=\operatorname{diag}\left[\Lambda_{j}\right]$ and $\Lambda_{j}$ is the Jordan block associated with the pole $z_{j}$ with multiplicity of $m_{j}$. The similarity transformation $\mathbf{J}=P^{-1} \mathbf{A}_{\mathbf{d}} P$ transforms the discrete system matrix $\mathbf{A}_{\mathbf{d}}$ into Jordan canonical form $\mathbf{J}$, which is nearly a diagonal matrix. The columns of transformation matrix $\mathrm{P}$ are the eigenvectors of $\mathbf{A}_{\mathbf{d}}$. The control influence matrix $\mathbf{B}_{\mathbf{d}}$ and the end conditions are transformed accordingly.

$$
\Gamma=P^{-1} \mathbf{B}_{\mathbf{d}}, \underline{x}(0)=P^{-1} \underline{y}(0), \underline{\mathrm{x}}(N T)=P^{-1} \underline{y}(N T)
$$

As an example, consider a Jordan block $\Lambda_{1}$ defined above) associated with a pole $z_{1}$ which has a multiplicity of four, so the state equation is

$$
\underline{x}[(k+1)]=\Lambda_{1} \underline{x}(k)=\left[\begin{array}{cccc}
z_{1} & 1 & 0 & 0 \\
0 & z_{1} & 1 & 0 \\
0 & 0 & z_{1} & 1 \\
0 & 0 & 0 & z_{1}
\end{array}\right] \underline{x}(k)
$$

It is possible to find the state transition matrix in a systematic manner with almost the same ease as in the case of a diagonal matrix. Notice that the last state equation in Eq. (8) is entirely decoupled from the other equations, thus the fourth element of the vector $\underline{x}$ can be found as $x_{4}(k)=z_{1}^{k} x_{4}(0)$. Substituting $x_{4}(k)$ in the third state equation, solve for $x_{3}(k)$

$$
x_{3}(k+1)=z_{1} x_{3}(k)+x_{4}(k)=z_{1} x_{3}(k)+z_{1}^{k} x_{4}(0)
$$

Eq. (9) can now easily be solved for $x_{3}$ :

$$
x_{3}(k)=z_{1}^{k} x_{3}(0)+k z_{1}^{k-1} x_{4}(k)
$$

Continuing with the same process, the solution of $x_{1}(k)$ is

$$
x_{1}(k)=z_{1}^{k} x_{1}(0)+\ldots+\frac{k(k-1)(k-2)}{3 !} z_{1}^{k-3} x_{4}(0)
$$

In matrix form, the state transition equation is written as $\underline{x}(k)=J_{1}^{k} \underline{x}(0) \quad$ where

$$
J_{1}^{k}=z_{1}^{k}\left[\begin{array}{cccc}
1 & k z_{1}^{-1} & \frac{k(k-1)}{2 !} z_{1}^{-2} & \frac{k(k-1)(k-2)}{3 !} z_{1}^{-3} \\
0 & 1 & k z_{1}^{-1} & \frac{k(k-1)}{2 !} z_{1}^{-2} \\
0 & 0 & 1 & k z_{1}^{-1} \\
0 & 0 & 0 & 1
\end{array}\right]
$$

Using the same recursive approach, the solution of Eq. (6) can be derived as

$$
\underline{x}(N T)=\mathbf{J}^{N} \underline{x}(0)+\sum_{k=0}^{N-1} \mathbf{J}^{N-k-1} \Gamma \underline{u}(k T)
$$

where, $\mathbf{J}^{N}=\operatorname{diag}\left[\Lambda_{j}^{N}\right]$, and

$$
\Lambda_{j}^{N}=z_{j}^{N}\left[\begin{array}{cccc}
1 & N z_{j}^{-1} & \ldots & \frac{N(N-1) \cdots\left(N-m_{j}+2\right)}{\left(m_{j}-1\right) !} z_{j}^{-m_{j}+1} \\
0 & 1 & \ldots & 0 \\
0 & 0 & \ddots & 0 \\
\vdots & \vdots & \ldots & \vdots \\
0 & 0 & \cdots & \frac{N(N-1)}{2 !} z_{j}^{-2} \\
0 & 0 & \ldots & N z_{j}^{-1} \\
0 & 0 & \ldots & 1
\end{array}\right]
$$

\section{Solution of the Discrete Open-Loop Control}

Under the assumption that the control input $u(k T)$ is represented as a linear combination of some independent components, the control input can be written as

$$
u(k T)=\underline{\phi}^{T}(K T) \underline{\lambda}
$$

where $\phi^{T}(K T)$ and $\underline{\lambda}$ are the assumed control profile independent variable vector and their coefficients respectively.Using Eq. (15), Eq. (13) can be rewritten as

$$
\underline{x}(N T)-\mathbf{J}^{N} \underline{x}(0)=Z(T, N) \underline{\lambda}
$$

where $Z(T, N)=\sum_{k=0}^{N-1} \mathbf{J}^{N-k-1} \Gamma \underline{\phi}^{T}(k T)$. For a control input $u(k T)$ as specified in Eq. (15) and a given end conditions $\underline{x}(N T)$, Eq. (16) can be solved for the unknown coefficients $\underline{\lambda}$

$$
\underline{\lambda}=Z^{-1}(T, N)\left[\underline{x}(N T)-\mathbf{J}^{N} \underline{x}(0)\right]
$$

\section{Optimal Solution of the Discrete Open-Loop Control \\ The optimal control problem is to find the control $\underline{u}_{k}^{*}$ on the interval $[0, N]$ that drives the system along a trajectory $\underline{x}_{k}^{*}$ such that a given performance index is}


minimized. Suppose we are concerned with minimizing the power of a single input problem, that is

$$
\begin{gathered}
J^{k}=\sum_{k=0}^{N-1} u^{2}(k T) \\
\text { s.t.: } \underline{x}[(k+1) T]=\mathbf{J} \underline{x}(k T)+\Gamma \underline{u}(k T)
\end{gathered}
$$

Because the constraint equation is specified at each time $\mathrm{k}$ in the interval of interest, a Lagrange multiplier is required at each time step, i.e. each constraint has an associated Lagrange multiplier $\left(\underline{\lambda}_{k} \in R^{n}\right)$. To find the optimal control profile $u(k)$ that drives the system from a given initial state $\underline{x}(0)$ to the desired $\underline{x}(N)$ over the interval $[0, N]$ while minimizing Eq. (18), define the Hamiltonian as

$$
H^{k}=u_{k}^{2}+\underline{\lambda}_{k+1}^{T}\left[\mathbf{J} \underline{x}_{k}+\Gamma u_{k}\right]
$$

the set of necessary conditions to be satisfied are given by (Lewis [7]).

$$
\begin{array}{ll}
\text { state equation: } & \underline{x}_{k+1}=\frac{\partial H^{k}}{\partial \underline{\lambda}_{k+1}}=\mathbf{J} \underline{x}_{k}+\Gamma u_{k} \\
\text { co-state equation: } & \underline{\lambda}_{k}=\frac{\partial H^{k}}{\frac{x_{k}}{x_{k}}=\mathbf{J}^{T} \underline{\lambda}_{k+1}} \quad(20) \\
\text { stationarity } & u_{k}=-\frac{1}{2} \Gamma^{T} \underline{\lambda}_{k+1}
\end{array}
$$

Eq. (20) are a set of linear homogeneous difference equations. We can rewrite the costate equations as

$$
\underline{\lambda}_{k}=\left(\mathbf{J}^{T}\right)^{m} \underline{\lambda}_{k+m}
$$

Let $m=N-k$, then the solution of $\underline{\lambda}_{k}$ can be represented by the final co-state vector $\underline{\lambda}_{N}$ as

$$
\underline{\lambda}_{k}=\left(\mathbf{J}^{T}\right)^{N-k} \underline{\lambda}_{N}
$$

Substituting Eq. (22) into Eq. (21) with $k=k+1$, solve for the optimal control profile in terms of the final costate vector as

$$
u(k)=-\frac{1}{2} \Gamma^{T}\left(\mathbf{J}^{T}\right)^{N-k-1} \underline{\lambda}(N)=\underline{\phi}^{T} \underline{\hat{\lambda}}_{N}
$$

\section{Sensitivity to System Parameters}

The design of accurate control system in the presence of significant uncertainty requires the designer to seek a robust system. The plant model will always be an inaccurate representation of the actual physical system because of parameter changes, unmodeled dynamics, time delays, sensor noise. The goal of robust system design is to retain assurance of system performance in spite of model inaccuracies and changes. If we want to reduce the influence of the uncertainty of the parameters on the final states, we can include the sensitivity to these parameters inside the state equations. From
Eq. (6), the relation of the pole $z_{j}$ associated with the state $x_{j}$ with multiplicity 1 in Jordan canonical form is

$$
x_{j}(k+1)=\left.z\right|_{z=z_{j}} x_{j}(k)+\gamma_{j} u(k)
$$

Differentiating Eq. (24) with respect to $z$, the sensitivity equation is obtained as

$$
\frac{d x_{j}(k+1)}{d z}=x_{j}(k)+\left.z\right|_{z=z_{j}} \frac{d x_{j}(k)}{d z}
$$

assuming new state $x_{n+j}(k)=d x_{j}(k) / d z$, then Eq. (25) becomes

$$
x_{n+j}(k+1)=x_{j}(k)+z_{j} x_{n+j}(k)
$$

the new discrete state equation is similar to Eq. (6) and the combined system can be represented as

$$
\underline{\hat{x}}(k+1)=\hat{\mathbf{J}} \underline{\hat{x}}(k)+\hat{\Gamma} \underline{u}(k)
$$

where,

$$
\hat{\mathbf{J}}=\left[\begin{array}{cccccc}
z_{1} & 1 & 0 & 0 & 0 & 0 \\
0 & z_{1} & 0 & 0 & 0 & 0 \\
0 & 0 & z_{2} & 0 & 0 & 0 \\
0 & 0 & 0 & z_{3} & 0 & 0 \\
0 & 0 & 1 & 0 & z_{2} & 0 \\
0 & 0 & 0 & 1 & 0 & z_{3}
\end{array}\right]
$$

and $\quad \underline{\hat{x}}(k)=\left[\underline{x}(k)_{n \times 1}\left(\frac{d x_{j}(k)}{d z}\right)_{j \times 1}\right]^{T}, \hat{\Gamma}=\left[\begin{array}{ll}\Gamma_{n \times 1} & \underline{0}_{3 \times 1}\end{array}\right]^{T}$, applying similarity transformation, the Jordan canonical form of Eq. (27) and the corresponding optimal and sub-optimal control profiles can be found using the same procedure outlined above.

\section{Simulation and Numerical Examples}

A systematic procedure to obtain the control profile for a system undergoing a rest-to-rest maneuver in discrete time domain is presented in this section, the results are then compared with those of the corresponding continuous system. The solution for the control parameters is obtained for a plant with a repeated poles using various sampling periods. Both sub-optimal and optimal solutions are presented. The effect of the uncertainty in the system's parameters is addressed by introducing the sensitivity equations and the optimal control profile for this case is derived. The following general procedure is adapted throughout the this paper : 1 . Check the rank of the system controllability matrix. It must be fully controllable system, 2 . Discretize the system using Eq. (4), 3. Find the Jordan canonical form of the discrete state equation as shown in Eq. (6) and the corresponding generalized eigenvectors $P$ associated with it, 4. Transform the system control influence matrix $\hat{\mathbf{B}}$ and the boundary conditions using Eq. (7), 5. Assume a control profile $u(k T)=\phi^{T}(K T) \underline{\lambda}$, which is a vector of linearly independent components and use Eq. (17) to solve for $\underline{\lambda}$. 
Example 1: Consider the continuous time transfer function $Y(s) / U(s)=1 / s^{2}\left(s^{2}+1\right)$ s.t. $: y\left(t_{f}\right)=1$, and all other end conditions are zero at $t_{i}=-4 \pi$ and $t_{f}=0$. This is an undamped system with one rigid body mode and one flexible mode and natural frequency of $1 \mathrm{rad} / \mathrm{sec}$. Using Eq. (6) for a sampling period of $0.1 \mathrm{sec}$, the equivalent Jordan Canonical form of the system

$$
J=\left[\begin{array}{cccc}
.9950+.0998 \imath & 0 & 0 & 0 \\
0 & .9950-.0998 \imath & 0 & 0 \\
0 & 0 & 1 & 1 \\
0 & 0 & 0 & 1
\end{array}\right]
$$

and the new control influence matrix and end conditions are respectively: $\Gamma=\left[\begin{array}{ll}.099+.005 \imath & .099-\end{array}\right.$ $.005 \imath \quad .050 \quad .100]^{T}, x(0)=\left[\begin{array}{llll}0 & 0 & 0 & 0\end{array}\right]^{T}, x(N)=$ $\left[\begin{array}{llll}0 & 0 & 10 & 0\end{array}\right]^{T}$. Assuming the control profile has a polynomial form given by $u(k T)=\phi^{T}(K T) \underline{\lambda}$, where $\quad \phi^{T}(k T)=\left[\begin{array}{llll}1 & (-k T) & (-k T)^{2} & (-k T)^{3}\end{array}\right]$. Solving for the control profile parameters using Eqs. (15), (16) and (17), we have $\underline{\lambda}=-\left[\begin{array}{llll}0.0188 & 0.0501 & .0114 & 0.0006\end{array}\right]$, and the associated control profile is

$$
u(k)=-0.019+0.050(k)-0.011(k)^{2}+0.0006(k)^{3}
$$

To find the optimal control profile, the control profile is assumed to be a function of the systems eigenvalues. Because we have a repeated poles, the equivalent transformation must be done by introducing the generalized eigenvectors as shown in $\mathrm{Eq}$. (23): and the corresponding control profile is

$$
\begin{aligned}
& u(k)=\left[\begin{array}{ll}
z_{1}^{N-1-k} & (N-1-k) z_{1}^{N-2-k}
\end{array}\right. \\
& \left.z_{2}^{N-1-k} z_{3}^{N-1-k}\right]\left[\lambda_{i}\right]^{T}
\end{aligned}
$$

where $z_{1}$ is the repeated pole. For this system, the vector of the optimal control profile parameters $\underline{\lambda}$ can be evaluated by Eq. (17) as

$$
\underline{\lambda}=10^{-2}\left[\begin{array}{llll}
-4.4 & .07 & .02+.7 \imath & .02-.7 \imath
\end{array}\right]^{T}
$$

For this sampling period the control profile is

$$
u(k)=\lambda_{0} \times z_{1}^{p}+\lambda_{1} p \times z_{1}^{p-1}+\lambda_{2} \times z_{2}^{p}+\lambda_{3} \times z_{3}^{p}
$$

where $p=N-1-k$ and $N$ is the number of sampling periods. The system response for the control input given by Eq. 30 is shown in Fig. (1). To compare the discrete system results with the continuous system response, the simulations for the system given in [4] are also presented in the same figure. Plots of the optimal control profile and the system response are shown in Figs. (1) and (2).

Example 2: To examine the robustness of the system response to the parameter uncertainty in the flexible mode poles $z_{2}$ and $z_{3}$, the same system as considered previously will be discussed here, this time with the
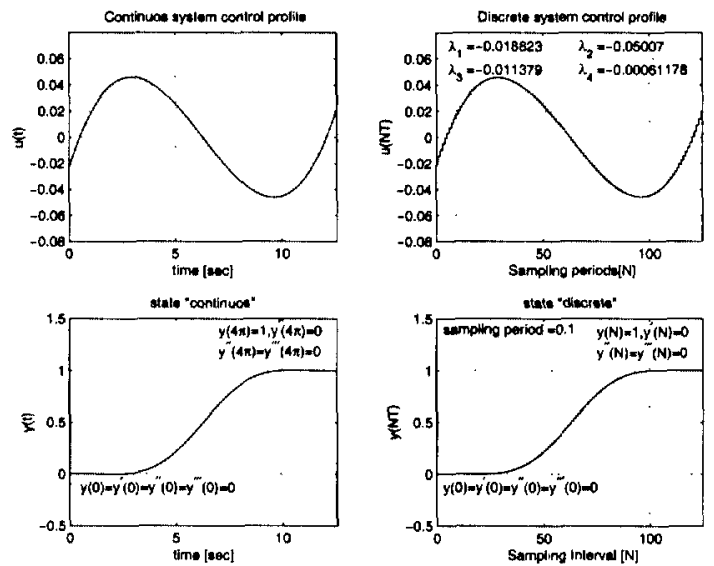

Figure 1: open-loop control of example 2 using polynomial input, $T=0.1$ sec.
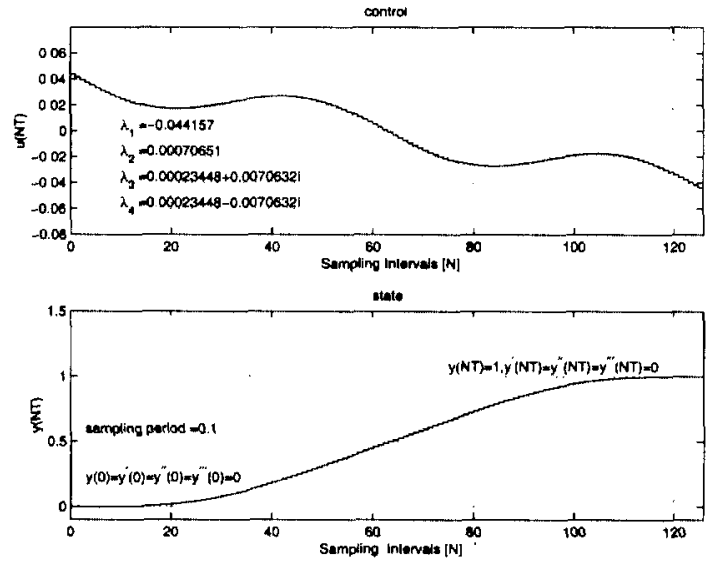

Figure 2: minimum power control and system response of example $2, T=0.1 \mathrm{sec}$.

inclusion of sensitivity equations. As in the previous example, a sampling period of $0.1 \mathrm{sec}$ is used. Use Eq. (27) and similarity transformation to examine the influence of parameter uncertainty of the flexible mode poles $z_{2}$ and $z_{3}$. The resulting Jordan canonical for this system has 3 poles each of multiplicity 2 . Following the same procedure of the previous example, the optimal control profile is assumed to be

$$
\begin{aligned}
u(k) & =\lambda_{0} \times z_{1}^{p}+\lambda_{1} p \times z_{1}^{p-1}+\lambda_{2} \times z_{2}^{p}+\lambda_{3} p \times z_{2}^{p-1} \\
& +\lambda_{4} \times z_{3}^{p}+\lambda_{5} p \times z_{3}^{p-1}
\end{aligned}
$$

where, $p=N-1-k$ and $z_{i}, \lambda_{i}$ are respectively the elements of

$$
\begin{gathered}
\underline{\phi}(k)=\left[\begin{array}{ll}
z_{1}^{N-1-k} & (N-1-k) z_{1}^{N-2-k} \\
z_{2}^{N-1-k} & (N-1-k) z_{2}^{N-2-k} \\
z_{3}^{N-1-k} & (N-1-k) z_{3}^{N-2-k}
\end{array}\right]^{T} \\
\underline{\lambda}=\left[\begin{array}{rrr}
-4.57 e^{-2} & 7.31 e^{-4} & 5.61 e^{-3}+6.70 e^{-3} \imath \\
-8.56 e^{-5}+1.14 e^{-5} \imath & 5.61 e^{-3}-6.70 e^{-3} \imath \\
-8.56 e^{-5}-1.14 e^{-5} \imath
\end{array}\right]^{T}
\end{gathered}
$$


Plots of the optimal control profiles with and without considering the sensitivity equations are shown in Fig. (3) for a sampling period $T=0.1 \mathrm{sec}$ and natural frequency of $1 \mathrm{rad} / \mathrm{sec}$. In Figs. (4), a system with natural frequencies $\pm 5 \%$ of the original is subjected to the same optimal control input. It shows that the system performance is robust to the model inaccuracies and changes. The system response at the final state for each frequency is zoomed up on the right subplots.

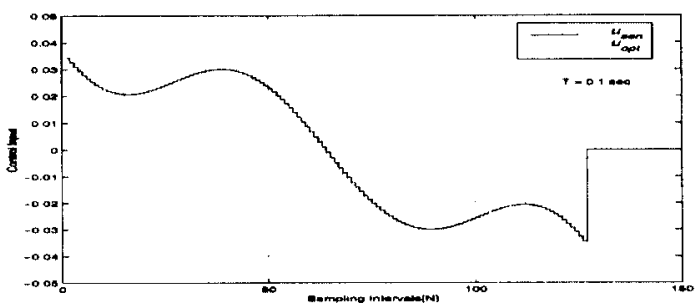

Figure 3: minimum power control profiles of example 2 with sensitivity, sampling period $T=0.1 \mathrm{sec}$.
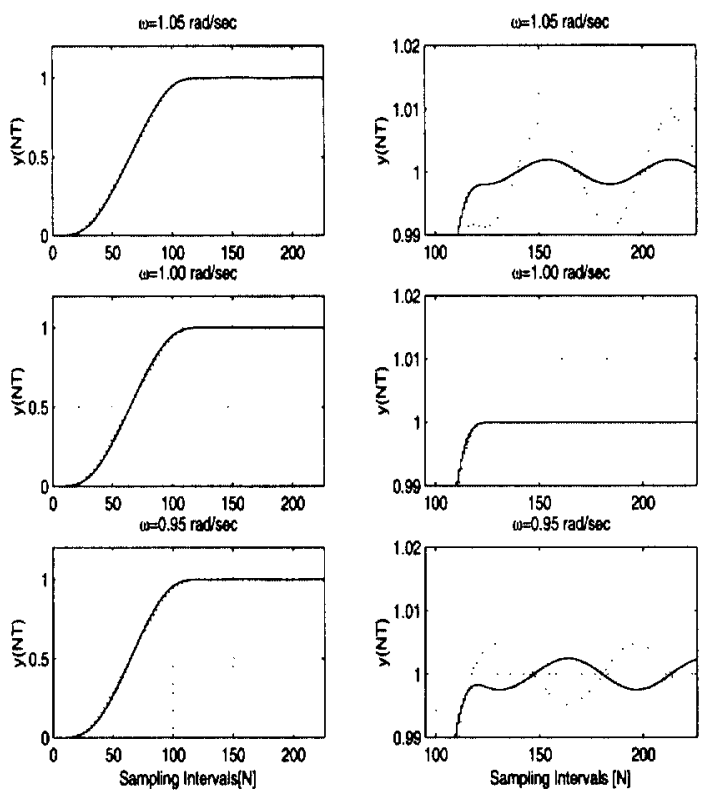

Figure 4: system response of example 2 with variation of natural frequency $\pm 0.05 \mathrm{rad} / \mathrm{sec}$.

\section{Conclusion}

The focus of this paper was on solving the point to point control problem in discrete time domain and compare it with the results obtained by (Miu \& Bhat [5]) and other researchers for continuous time systems. They used a finite Laplace transform technique for solving the benchmark problem with spring-mass system in continuous time. The same example was used in this study in order to compare the control results in discrete time domain. The original continuous system were discretized and decoupled by using the similarity transformation which change the difference equations into a set of algebraic equations that can be easily solved.

The finite time Laplace transform used in continuous time system by (Miu \& Bhat [5]) was replaced by a method which can be called a finite $\mathrm{Z}$ transform. For this class of problems, the results obtained were compatible with the continuous time counterparts. As the sampling period was decreased, the behavior of the system approaches that of the continuous time system. The shape of control profile depends on the position of the poles, which in turn depends on the sampling period used.

The sensitivity analysis shown in example 2, illustrates that the added states based on the parameters of the system can significantly reduce the influence of uncertainty of identification of the parameters. The proposed control system exhibits the desired performance despite the presence of plant uncertainty.

\section{References}

[1] Juang J.N. et al, Closed form solution of for Feedback Problems with Terminal Constraints, Journal of Guidance and Control Vol. 8,,No. 1 Jan-Feb, pp. 127-135, 1985.

[2] Singh, G. Kabama,et al, Planar, Time-Optimal - Rest-to-Rest Slewing Maneuver of Flexible Space craft,Journal of Guidance and Control Vol. 12,,No. 1 pp.71-81, 1989. Theory of Machines and Mechanisms, Vol.3, pp. 1489-1494, 1987.

[3] Cannon, R. H.,et al, Initial Experiments on the End Point Control of Flexible one Robot, International J. of Robotics Research, Vol. 3, pp 62-75, 1984.

[4] Miu, D. K., Bhat S. P., Precise Point to Point Positioning Control of Flexible Structures, ASME J. of Dynamic Systems, Measurement, and Control, Vol. 112, pp. 667-674, 1990 .

[5] Miu, D. K., Bhat S. P., Solutions of Point to point Control Problems Using Laplace Transform Technique, ASME J. of Dynamic Systems, Measurement, and Control, Vol. 113, pp. 425-431, 1991.

[6] Kuo, B.C., Digital Control Systems,Saunders College Publishing. 1992.

[7] Lewis, F. L.,Syrmos, V. L., Optimal Control, 2nd edition. John Wiley \& Sons, Inc. 1995. 